FORMATION Formation emploi

Revue française de sciences sociales

118 | avril-juin 2012

10 ans de parcours professionnels des jeunes :

l'intérêt des études longitudinales

\title{
Faut-il tourner la PAJE ? : l'impact de la Prestation d'Accueil du Jeune Enfant sur le parcours professionnel des mères
}

Turning the PAJE: the impact of the Prestation d'Accueil du Jeune Enfant on the mother's professional career

Berufliche Segregation und Entlohnung am Laufbahnbeginn: einige Berufe unter der Lupe

“Hay que dar vuelta la PAJE ?” : el impacto de la Prestation d'Accueil du Jeune Enfant (prestación de recepción del niño) sobre el itinerario profesional de las madres

Olivier Joseph, Ariane Pailhé, Isabelle Recotillet et Anne Solaz

\section{(2) OpenEdition \\ Journals}

Édition électronique

URL : http://journals.openedition.org/formationemploi/3642

DOI : $10.4000 /$ formationemploi.3642

ISSN : 2107-0946

Éditeur

La Documentation française

Édition imprimée

Date de publication : 30 juin 2012

Pagination : 103-123

ISSN : 0759-6340

Référence électronique

Olivier Joseph, Ariane Pailhé, Isabelle Recotillet et Anne Solaz, « Faut-il tourner la PAJE ? : I'impact de la Prestation d'Accueil du Jeune Enfant sur le parcours professionnel des mères », Formation emploi [En ligne], 118 | avril-juin 2012, mis en ligne le 23 juillet 2012, consulté le 30 octobre 2020. URL : http:// journals.openedition.org/formationemploi/3642; DOI : https://doi.org/10.4000/formationemploi.3642 


\title{
Faut-il tourner la PAJE ? :
}

\section{L'impact de la Prestation d'Accueil du Jeune Enfant sur le parcours professionnel des mères}

\author{
Olivier JOSEPH \\ Économiste, chargé d'études au Céreq \\ Ariane PAILHÉ \\ Économiste, directrice de recherche à l'INED, Institut national d'études démographiques \\ Isabelle RECOTILLET \\ Économiste, chef du département entrées et évolutions dans la vie active au Céreq \\ Anne SOLAZ \\ Économiste, chargée de recherche à l'INED, Institut national d'études démographiques
}

Résumé

Faut-il tourner la PAJE ? : l'impact de la Prestation d'Accueil du Jeune Enfant sur le parcours professionnel des mères

Larrivée des enfants entraine souvent un retrait du marché du travail des femmes, favorisé par l'existence d'allocations de remplacement. Ce retrait, souvent temporaire, peut toutefois avoir des répercussions professionnelles. À partir de l'enquête du Céreq à dix ans auprès de la Génération 98, l'article évalue les conséquences professionnelles, pour les mères, de la réforme de la PAJE (Prestation d'Accueil du Jeune Enfant) qui, en 2004, a étendu la compensation financière de l'arrêt aux premières naissances. Depuis, davantage de mères d'un premier enfant interrompent totalement ou partiellement leur activité, pour une courte durée. Un impact négatif sur leur salaire est visible jusqu'à deux ans après la naissance.

Mots clés : Femme $\bullet$ cheminement professionnel $\bullet$ politique familiale $\bullet$ enquête génération 1998

Abstract

Turning the PAJE: the impact of the Prestation d'Accueil du Jeune Enfant on the mother's professional career

Childbirth often means that women withdraw from the labour market, with incentive provided by replacement benefits. Such a withdrawal, while it is usually temporary, may 
nonetheless have repercussions on a woman's career. Based on the Céreq Génération 98 survey, this paper assesses how far mothers' careers are affected by the reform of the early childhood benefit, PAJE (Prestation d'Accueil du Jeune Enfant), which, in 2004, extended financial benefits provided under the Order to the birth of the first child. Since then, increasing numbers of first-time mothers have taken a total or partial break from work for a short period. It has been found that this can have a negative impact on their salaries for up to two years following the birth.

Keywords: Woman $\bullet$ occupational paths $\bullet$ family policy $\bullet$ generation 98 survey Journal of Economic Literature: J 24, J 13

Traduction : Provence Traduction

La publication quasi simultanée des rapports de l'OCDE (Organisation de coopération et de développement économiques) sur les déterminants du bien-être des familles (OCDE, 2011) et de l'IGAS (Inspection générale des affaires sociales) sur l'égal accès des femmes et des hommes aux responsabilités professionnelles et familiales dans le monde du travail (Grésy et al., 2011) a relancé le débat sur la durée des congés parentaux en France. En effet, ces deux rapports soulignent que de trop longues interruptions d'activité pénalisent l'ensemble de la trajectoire professionnelle des femmes : elles impliquent à la fois de moindre chances de retour à l'emploi, des profils de rémunération plus plats, un moindre avancement et des niveaux de retraite plus faibles. Les interruptions de carrière liées à la maternité figurent parmi les principaux facteurs explicatifs des moindres salaires perçus par les femmes (Meurs et al., 2011 ; Ruhm, 1998 ; Jaumotte, 2003 ; Lequien, 2012). Ces interruptions réduisent l'expérience professionnelle (Becker, 1964), déprécient le capital humain (Mincer et Polachek, 1974), ou peuvent encore être interprétées par les employeurs comme un "signal " d'un moindre engagement professionnel (Albrecht et al., 1999). Afin de moins pénaliser les carrières des femmes, et de permettre à plus de parents de prendre un congé, ces rapports recommandent une réforme du congé parental qui consiste à réduire sa durée à un an maximum et à mieux le rémunérer.

Des incitations à de courtes interruptions d'activité existent déjà depuis 2004 ; elles concernent uniquement les premières naissances. La dernière réforme du dispositif d'indemnisation des interruptions de carrière pour s'occuper d'enfant a ainsi introduit la possibilité de bénéficier d'une allocation, le complément libre choix d'activité (CLCA), dès le premier enfant, pour une durée maximale de six mois, à taux plein ou partiel. Avant 2004, les mères d'un premier enfant pouvaient prendre un congé parental de trois ans, garantissant le retour à l'emploi dans la même entreprise, mais ne percevaient aucune compensa- 
tion en contrepartie. L'objectif de ce travail est d'évaluer l'impact de cette réforme et, plus généralement, l'influence de la prise d'un congé parental court sur la trajectoire professionnelle des mères d'un enfant ${ }^{1}$. Cette réforme a-t-elle incité les jeunes femmes actives sur le marché du travail à cesser leur activité momentanément ou à la réduire ? Quel a été l'impact de cette interruption de carrière sur la suite de leur parcours professionnel, en particulier en termes de salaire et de participation au marché du travail ?

Plusieurs travaux ont déjà évalué l'impact de réformes de l'indemnisation des interruptions de carrière pour s'occuper d'enfants en France. Ils se sont intéressés à la précédente réforme, datant de 1994, qui avait rendu possible la perception de l'allocation parentale d'éducation (APE) pour la naissance d'un deuxième enfant pour une durée de trois ans (seuls les parents d'au moins trois enfants en étaient bénéficiaires auparavant). Ces travaux ont montré que cette réforme a incité un grand nombre de jeunes mères à interrompre leur carrière dans les trois années suivant la naissance de leur deuxième enfant (Lequien, 2012 ; Piketty, 2005) avec un effet négatif sur leurs salaires ultérieurs.

La réforme de 2004 a introduit une indemnisation des interruptions d'activité pour les premières naissances, mais relativement courte (6 mois, contre 3 ans pour les naissances suivantes). Nous cherchons ici à évaluer dans quelle mesure une interruption plus courte peut également induire un effet pénalisant sur les carrières des femmes. Ce travail pourra alimenter une réflexion plus large autour d'une réforme générale de la politique d'indemnisation du congé parental. En effet, les travaux empiriques soulignent que les interruptions courtes dans la carrière ne produisent pas d'effet négatif sur les carrières, contrairement aux interruptions longues; cependant, une réforme de la politique familiale pourrait consister à proposer des congés parentaux plus courts mais mieux indemnisés.

À cet effet, nous mobilisons les données de la quatrième interrogation de l'enquête Génération 98 réalisée par le Céreq. Elle permet d'étudier des trajectoires individuelles sur dix années de vie active. Cette enquête comporte également des informations sur les naissances et l'interruption d'activité, totale ou partielle, après chacune d'entre elles.

Dans un premier temps, nous présentons les principes de la réforme et les modalités de l'indemnisation des interruptions d'activité pour s'occuper de jeunes enfants en France. Puis une description des bénéficiaires du CLCA au premier enfant (rang 1) est réalisée, avant de présenter la méthode et les résultats d'estimation de l'évaluation de l'effet de la réforme sur les salaires et la participation au marché du travail.

1. Sachant que $98 \%$ des bénéficiaires du CLCA de rang 1 sont des femmes, c'est leur situation que nous allons étudier ici. 


\section{Encadré 1}

Les conditions d'éligibilité à la PAJE (prestation d'accueil du jeune enfant) - CLCA (complément libre choix d'activité pour premier enfant)

Pour être éligible au CLCA pour premier enfant, il faut justifier d'au moins huit trimestres de cotisations vieillesse validés au titre d'une activité professionnelle, dans les deux années civiles précédant la naissance ou l'adoption. Cette condition ne signifie pas qu'il faut nécessairement avoir travaillé au cours des huit trimestres précédant la naissance. Pour valider un trimestre, il faut avoir perçu, au cours de l'année, un salaire correspondant à 200 fois le SMIC (salaire minimum interprofessionnel de croissance) horaire en vigueur l'année considérée $(*)$. Pour valider quatre trimestres, il suffit d'avoir un salaire annuel équivalant à 800 fois le SMIC horaire, soit approximativement $40 \%$ du SMIC annuel $\left(^{* *}\right)$. Une activité à mi-temps rémunérée au SMIC permet donc de valider quatre trimestres. Les périodes de maternité et de congé maladie sont assimilées à une activité professionnelle, mais les périodes de chômage indemnisé et de formation professionnelle rémunérées ne peuvent être validées pour le CLCA de rang 1 . Il a été estimé que ces conditions d'activité antérieures sont assez restrictives; elles ont ainsi exclu $32 \%$ des mères d'un premier enfant (HCF, 2009).

$\left({ }^{*}\right)$ : Le salaire de référence pour la validation d'un trimestre est en effet fixé à 1438 euros, ce montant correspondant au salaire versé pour 200 heures travaillées au Smic.

$\left.{ }^{(* *}\right)$ On ne peut valider plus de quatre trimestres par an.

\section{Le succès de la PAJE-CLCA pour un premier enfant}

\subsection{La réforme de la PAJE en 2004}

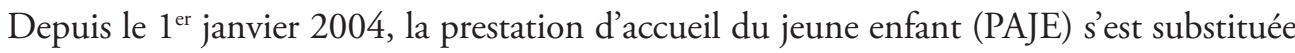
à l'ensemble des prestations accordées pour la naissance et la garde de jeunes enfants. La PAJE se décline en plusieurs volets. Parmi ceux-ci, le complément libre choix d'activité (PAJE-CLCA) est une prestation dont bénéficient les parents qui cessent (CLCA à taux plein) ou réduisent (CLCA à taux réduit) leur activité professionnelle pour s'occuper de leur enfant en bas âge. Cette prestation, versée par les caisses d'allocations familiales, s'est substituée à l'allocation parentale d'éducation (APE) ; cependant, contrairement à cette dernière, elle est attribuable dès le premier enfant, pour une durée de six mois maximum à compter de la naissance ou de la fin du congé de maternité, de paternité ou d'adoption. Pour les parents de deux enfants et plus, la durée de versement de l'allocation est inchangée par rapport à l'APE, la PAJE pouvant être versée jusqu'aux trois ans de l'enfant. La perception de cette prestation est soumise à des conditions d'éligibilité, qui dépendent de l'activité professionnelle passée. Ces conditions varient selon le rang de l'enfant, et sont relativement restrictives pour une première naissance (encadré $\mathbf{1}$ ). 
Tableau 1

Nombre de familles de un enfant bénéficiaires de la PAJE-CLCA

France entière - au 31 décembre 2009

\begin{tabular}{|l|c|c|c|c|c|c|}
\hline & 2004 & 2005 & 2006 & 2007 & 2008 & 2009 \\
\hline Taux Plein & 19948 & 21616 & 24990 & 22151 & 22086 & 21352 \\
\hline Taux Réduit 1 (Activité =>50\% à <=80\%) & 8038 & 10513 & 10740 & 11501 & 12056 & 12665 \\
\hline Taux Réduit 2 (Activité <50\%) & 3392 & 3519 & 3094 & 3099 & 3163 & 2918 \\
\hline Couples (les deux parents bénéficiaires) & 80 & 117 & 104 & 111 & 145 & 125 \\
\hline Total & 31458 & 35765 & 38928 & 36862 & 37450 & 37060 \\
\hline
\end{tabular}

Source : Données Cnaf.

Le montant du CLCA est forfaitaire, sans condition de ressources, et ne varie pas selon le rang de l'enfant. Il s'élève, en 2010 , à $552 €^{2}$ pour une allocation à taux plein, à $420 €$ pour un CLCA à taux partiel lorsque le temps de travail est inférieur à $50 \%$ de la durée du travail fixée dans l'entreprise, et à $317 €$ pour un CLCA à taux partiel si le temps de travail s'établit entre $50 \%$ et $80 \%$ de la durée du travail ${ }^{3}$. Le CLCA à taux partiel n'implique donc pas nécessairement une diminution des revenus, si l'allocation couvre la perte de salaire liée à la réduction du temps de travail.

Il est à noter que cette prestation, qui relève du droit de la sécurité sociale, se distingue du congé parental, qui lui relève du droit du travail. Tout parent justifiant d'un an d'ancienneté chez son employeur à la naissance de l'enfant (quel que soit son rang) a le droit de cesser son activité ou de travailler à temps partiel jusqu'au troisième anniversaire de l'enfant. À l'issue de ce congé, le retour à l'emploi précédent ou à un emploi similaire, assorti d'une rémunération équivalente, est garanti. Les conditions d'éligibilité au CLCA et au congé parental étant différentes, ces deux dispositifs ne se recouvrent pas complètement. Certains salariés peuvent être en congé parental sans bénéficier du CLCA et inversement.

\subsection{Un rapide succès du nouveau dispositif}

Cette nouvelle prestation a connu un rapide succès auprès des parents d'un enfant : chaque année, environ 37000 familles sont bénéficiaires du CLCA de rang 1 (tableau 1). Ces

2. Ce montant inclut l'allocation de base de la prestation d'accueil du jeune enfant pour ceux qui en bénéficient.

3. Par ailleurs, les deux membres du couple parental ne peuvent cumuler deux CLCA à taux plein ; mais ils peuvent tous deux percevoir le CLCA à taux partiel. 


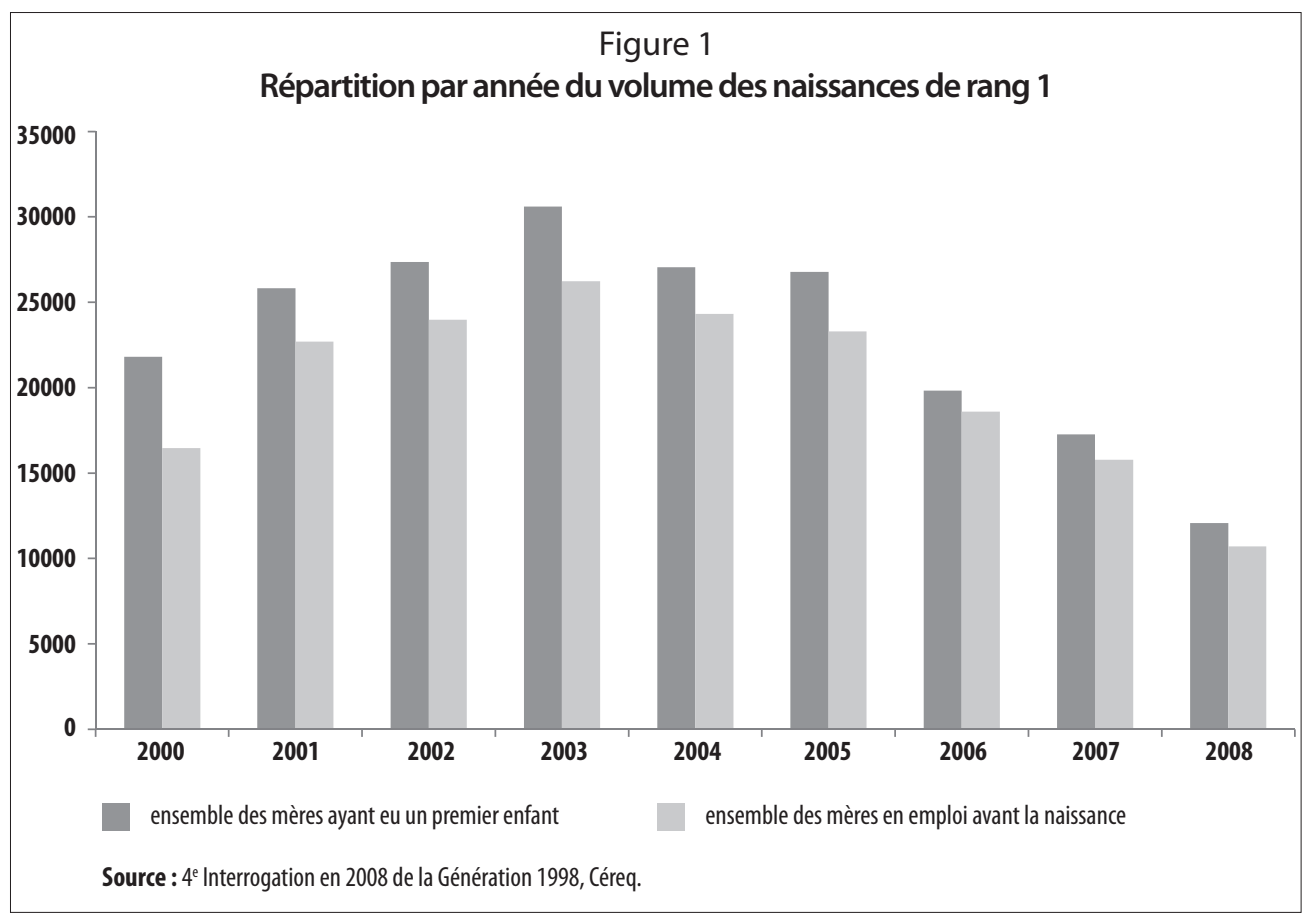

familles représentent près d'un tiers de l'ensemble des entrées dans le dispositif du CLCA (HCF, 2009). Les mères forment la quasi-totalité des bénéficiaires (97,6 \%), et la majorité d'entre elles (61\%) vont jusqu'au terme réglementaire de la prestation (Nicolas, 2010) ; la durée moyenne de perception de l'allocation est de 5,145 mois (HCF, 2009). Nous allons étudier ici quel a été l'impact de cette réforme sur les carrières des femmes qui ont interrompu leur activité.

\section{E) Éligibilité et accès au dispositif CLCA}

L'enquête Génération 98 a interrogé un échantillon représentatif des sortants du système éducatif en 1998, tous diplômes ou niveaux d'éducation confondus. Ces jeunes sortants ont été interrogés à plusieurs reprises, successivement en 2001, 2003, 2005 et 2008. Nous utilisons ici l'échantillon de femmes enquêtées et interrogées dix ans après la sortie du système éducatif, c'est-à-dire en $2008^{4}$.

4. Pouruneprésentation détailléedel'enquêteet deses différentesinterrogations, voirhttp://www.cereq.fr/index. php/sous-themes/Enquetes-Generation-Sous-Themes/Generation-1998-Enquetes-2001-2003-2005-2008 


\section{Encadré 2}

\section{Repérage du congé parental et calcul du critère d'éligibilité au CLCA (complément libre choix d'activité pour premier enfant) dans l'enquête Génération 98}

1. Les données de la 4 e interrogation de l'enquête Génération 98 offrent une perspective inédite pour évaluer la réforme de la PAJE (prestation d'accueil du jeune enfant) en 2004, pour au moins deux raisons. D'une part, la période d'observation des trajectoires individuelles est de longueur égale avant et après la réforme. Ensuite, contrairement aux données administratives de la Cnaf (Caisse nationale d'allocations familiales) qui portent uniquement sur la population des bénéficiaires, l'information contenue dans les données individuelles du Céreq sur les salaires, pour chacun des emplois occupés, permet de définir avec précision la population des mères éligibles au dispositif. Les données offrent une description détaillée sur les dix premières années de vie active du parcours professionnel depuis la fin des études avec, pour chaque emploi occupé, ses caractéristiques en début et fin. Par ailleurs, un certain nombre de questions autour de la famille et de la naissance permettent de mesurer les conséquences, en termes d'emploi, de la naissance des deux premiers enfants. La question de la prise du congé parental et du passage à temps partiel est posée dans ce cadre pour les deux premières naissances, mais uniquement pour les jeunes femmes occupant un emploi avant la naissance.

2. Les données permettent également de disposer des salaires nets, primes incluses, pour le début et la fin de chaque emploi dans une même entreprise. Afin de pouvoir appliquer précisément le critère d'éligibilité déterminé par la Cnaf (encadré 1), il a été nécessaire de reconstruire un calendrier des salaires des personnes interrogées. Pour les emplois longs, les interrogations intermédiaires du panel ont permis d'affiner les approximations d'évolution du salaire. Cela a permis en outre de prendre en compte le temps de travail dans le calcul. Ensuite, la valeur du Smic (salaire minimum interprofessionnel de croissance) pour chacune des années données a permis d'établir le nombre de trimestres validés au titre des cotisations vieillesse, suivant le critère énoncé dans l'encadré 1, à savoir : pour valider quatre trimestres, il faut avoir un salaire annuel équivalant à 800 fois le Smic horaire. Le calcul de l'éligibilité n'a pu cependant se faire qu'à partir de l'année 2000, afin d'avoir suffisamment de trimestres observés pour calculer le nombre de trimestres validés. Les indicateurs d'éligibilité ne sont donc fournis que pour la période 2000-2008.

\subsection{La moitié des femmes a eu un premier enfant avant la réforme de la PAJE}

Sur les 363000 jeunes femmes de l'interrogation, en 2008, de la Génération 98, 66 \% ont eu au moins un enfant dans les dix ans qui ont suivi la fin de leurs études, alors qu'elles n'étaient que $35 \%$ à être devenues mères dans les cinq années après la fin des études (Recotillet, Rouaud, Ryk, 2011). Le volume des naissances de rang 1 est croissant jusqu'en 2003, puis amorce une légère baisse (figure 1). Entre 2000 et 2008, $56 \%$ des mères ont eu leur premier enfant avant 2004. Ce constat n'est pas seulement factuel. Il révèle que le calendrier des naissances est fortement lié à l'âge des personnes interrogées dans l'enquête 
Tableau 2

Taux de mères éligibles au CLCA par niveau d'éducation (en \%)

\begin{tabular}{|l|c|c|c|}
\hline Niveau d'éducation en $\mathbf{1 9 9 8}$ & entre $\mathbf{2 0 0 0}$ et $\mathbf{2 0 0 8}$ & entre 2000 et $\mathbf{2 0 0 3}$ & entre 2004 et $\mathbf{2 0 0 8}$ \\
\hline Aucun diplôme & $\mathbf{3 6}$ & 24 & 54 \\
\hline Cap-Bep $\left(^{*}\right)$ & 59 & 47 & 75 \\
\hline Bac professionnel ou technologique & 66 & 58 & 73 \\
\hline Bac général & 56 & 50 & 61 \\
\hline Bac +2 & 76 & 69 & 85 \\
\hline 2e cycle & 78 & 73 & 85 \\
\hline 3e cycles et écoles & 74 & 64 & 91 \\
\hline
\end{tabular}

Source : 4 e Interrogation en 2008 de la Génération 1998, Céreq.

Champ : ensemble des mères.

Note de lecture : 36 \% des mères sans diplôme sont éligibles au CLCA entre 2000 et 2008.

${ }^{*}$ ) respectivement certificat d'aptitude professionnelle ; brevet d'études professionnelles.

et s'avère donc corrélé également à leur niveau de diplôme à la sortie du système éducatif' Ainsi, parmi les $45 \%$ de jeunes femmes diplômées de l'enseignement supérieur, l'arrivée du premier enfant survient plus rapidement après la fin de leurs études; elle arrive ainsi avant 2004 pour $58 \%$ d'entre elles. Par ailleurs, cette répartition par année du volume des naissances indique que la structure des données est adaptée à notre problématique, puisque le volume des naissances avant et après la réforme de 2004 est relativement équilibré.

La majorité des enquêtées devenues mères sur la période 2000-2008 occupaient un emploi avant la naissance de leur enfant : $83 \%$ d'entre elles étaient en emploi juste avant. Leur taux d'emploi est donc élevé, même s'il varie sur la période étudiée. Il est d'autant plus fort que l'on s'éloigne de la fin des études. Après 2004, elles sont $90 \%$ à occuper un emploi avant la naissance de leur premier enfant contre $77 \%$ avant 2004.

\subsection{L'éligibilité au CLCA dépend fortement du niveau de diplôme}

Les conditions d'accès au CLCA étant soumises à des conditions d'activité professionnelle (encadré 1), on observe plus de femmes éligibles après 2004 qu'avant. Si $66 \%$ des jeunes mères sont éligibles au CLCA sur l'ensemble de la période 2000-2008 (encadré 2), quelle que soit l'année de naissance de l'enfant et quelle que soit leur situation professionnelle avant la naissance, elles sont $76 \%{ }^{6}$ à devenir éligibles lorsque leur premier enfant nait après 2004. On observe également plus de mères éligibles au CLCA parmi celles qui occu-

5. Les jeunes femmes diplômées de l'enseignement supérieur en 1998 ont en moyenne 24 ans, alors que celles qui ont quitté l'école avec un diplôme inférieur ou équivalent au bac ont en moyenne 20 ans.

6. $28 \%$ ne seraient pas éligibles au CLCA. Ce chiffre est assez proche de l'estimation faite par le Haut conseil à la famille (HCF2009), qui est de $32 \%$. 
paient un emploi avant la naissance, mais avec un taux d'éligibles qui varie peu en fonction de la période. Parmi celles-ci, $81 \%$ sont éligibles entre 2000 et 2008 ; ce taux étant sensiblement identique sur les deux sous-périodes qui nous intéressent, à savoir 2000-2004 et 2004-2008. Ce point est particulièrement intéressant pour l'évaluation du dispositif. En effet, le fait d'observer un taux d'éligibles proche entre les deux sous-périodes indique que pour les mères en emploi avant la naissance, l'éligibilité n'est pas liée au temps écoulé depuis la fin des études, ce qui valide la comparaison des « éligibles » avant et après 2004.

En revanche, le taux d'éligibles oscille fortement selon le niveau d'éducation des jeunes mères (tableau 3), de $36 \%$ pour les mères sans diplôme, à $74 \%$ pour les diplômées de $3^{e}$ cycle ou de grandes écoles, avec, du reste, une répartition par niveau d'éducation qui évolue significativement avant et après 2004, particulièrement pour les non-diplômées. Ce constat peut s'expliquer par la plus grande probabilité des peu diplômées à être en marge de l'emploi. Or les périodes de chômage et de précarité à la sortie du système éducatif diminuent les chances d'être éligible avant la première naissance, d'autant plus que celle-ci se produit à des âges jeunes en moyenne pour les peu éduquées.

\subsection{Une progression des interruptions d'activité après la réforme de la PAJE}

Avant la réforme de la PAJE en 2004, la part de femmes optant pour un congé parental à l'occasion de la naissance de leur premier enfant (voir encadré 2 sur le repérage des congés parentaux dans l'enquête) était relativement faible : seulement $7 \%$ des mères en emploi avant la naissance prenaient un congé parental (sans perception d'allocation). Après la mise en place de la réforme, elles étaient $27 \%$ à opter pour un congé parental, qu'il corresponde à une interruption complète de leur activité professionnelle (à taux plein) ou seulement partielle (occupation d'un emploi à temps partiel).

Tableau 3

Taux d'interruption par niveau d'éducation

\begin{tabular}{|l|c|c|}
\hline & $\begin{array}{c}\text { Part d'interruption à taux plein } \\
\text { (en \%) }\end{array}$ & $\begin{array}{c}\text { Part d'interruption à taux partiel } \\
\text { (en \%) }\end{array}$ \\
\hline Aucun diplôme ou 1er $^{\text {en }}$ diplôme professionnel & 16 & 18 \\
\hline Bac (prof., techno ou général) & 12 & 16 \\
\hline Bac +2 & 8 & 20 \\
\hline Diplôme supérieur à bac + & 8 & 17 \\
\hline Ensemble & $10\left({ }^{* *}\right)$ & $17\left(^{*}\right)$ \\
\hline
\end{tabular}

Source : $4^{e}$ Interrogation en 2008 de la Génération 1998, Céreq.

Champ : Ensemble des mères en emploi avant la naissance de leur premier enfant.

Note de lecture : $\left(^{*}\right)$ parmi l'ensemble des mères occupant un emploi avant la naissance de leur premier enfant, $17 \%$ ont opté pour un congé parental à taux partiel et $\left.{ }^{* *}\right) 10 \%$ à taux plein. 
Figure 2

Taux d'interruption d'activité selon l'année de naissance du premier enfant, par tranches de salaire

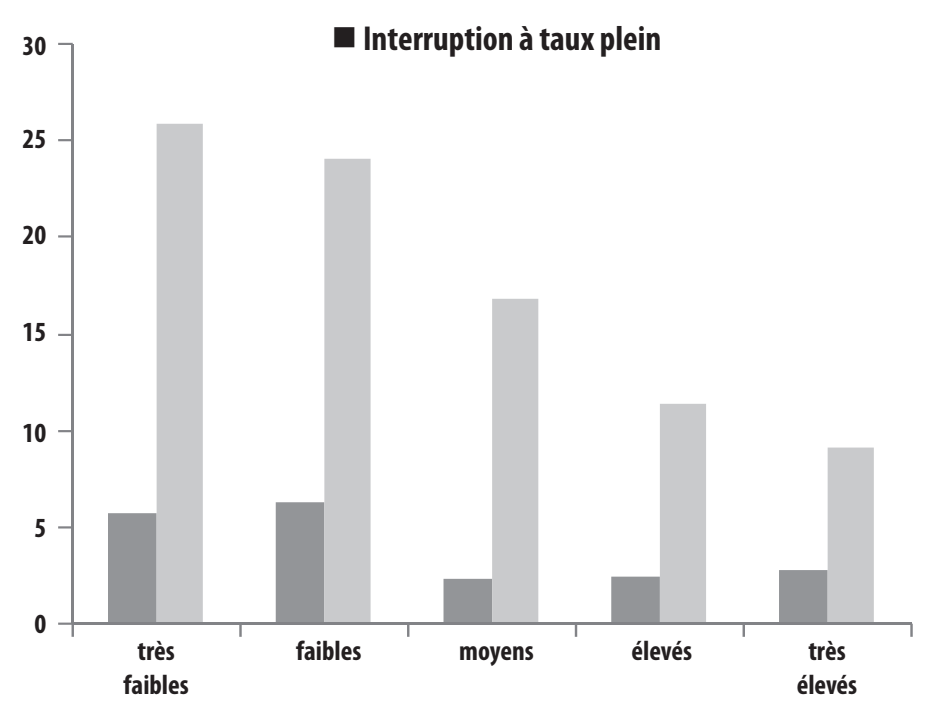

taux d'interruption à taux plein, avant 2004 taux d'interruption à taux plein, après 2004

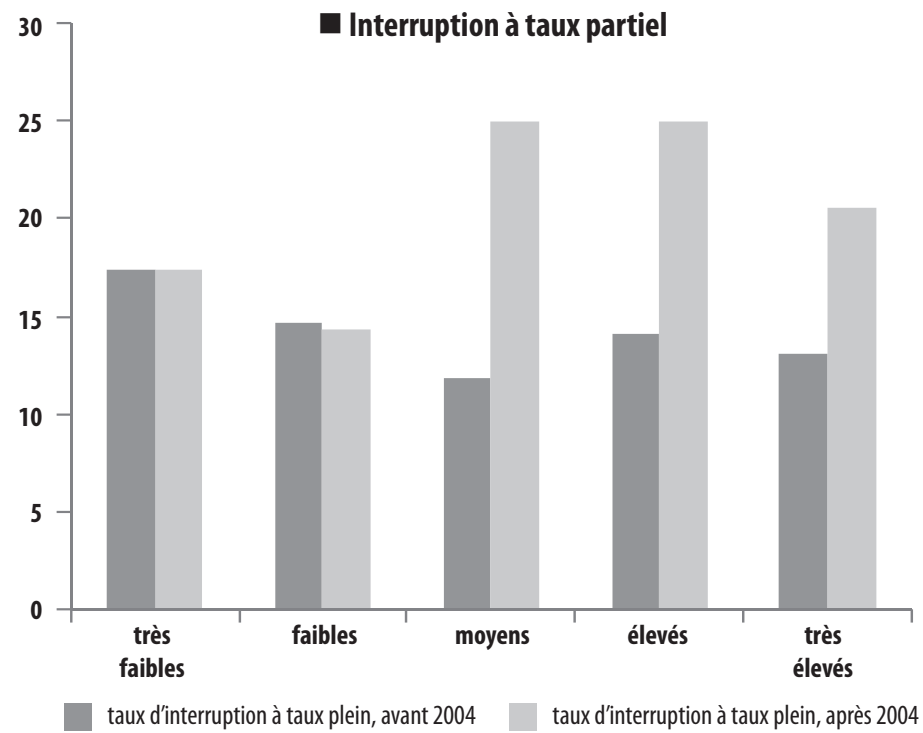

Source : $4^{e}$ Interrogation en 2008 de la Génération 1998, Céreq.

Champ : mères d'un premier enfant en emploi avant la naissance.

Note de lecture : Avant 2004, un peu plus de $5 \%$ des mères gagnant un très faible salaire ont interrompu totalement leur emploi suite à la naissance de leur premier enfant, tandis qu'après la réforme de 2004, elles sont un peu plus de $25 \%$. 
Que les jeunes mères soient éligibles ou non au CLCA, l'interruption de leur activité se réalise plus souvent à taux partiel (63\% des mères éligibles ont opté pour une interruption à taux partiel, $56 \%$ des non-éligibles). Par ailleurs, parmi celles qui choisissent de réduire partiellement leur activité, $60 \%$ l'ont fait lorsque leur premier enfant est né après 2004. Et parmi celles qui ont totalement interrompu leur activité, 82 \% l'ont fait après 2004.

Le CLCA, en distribuant un complément de revenu en contrepartie d'une interruption d'activité, a visiblement incité plus de jeunes mères à interrompre leur activité professionnelle, totalement ou partiellement. En offrant une compensation financière - même faible - à l'interruption de l'activité professionnelle, ce dispositif entraine une baisse du coût d'opportunité de cette interruption. Ce coût étant plus facile à supporter pour des interruptions partielles d'activité, on observe (tableau 3) que celles-ci sont plus fréquentes $(17 \%)$ que les interruptions totales (10 \%). Le coût d'opportunité de s'arrêter est généralement plus fort pour les jeunes mères les plus diplômées, qui sont aussi celles qui accèdent à des positions professionnelles et salaires plus élevés. Pour les femmes ayant poursuivi des études supérieures, les interruptions totales sont nettement moins fréquentes (8\%) et les interruptions partielles sont préférées.

Ces constats, dressés à partir des données de la 4e interrogation de la Génération 98, concordent avec l'essor observé - dans d'autres enquêtes - du CLCA à taux réduit auprès des classes moyennes et aisées. L'enquête menée par le Crédoc (Centre de recherche pour l'étude et l'observation des conditions de vie, la Cnaf et la Drees (Direction de la recherche, des études, de l'évaluation et des statistiques) auprès des allocataires de la PAJE, en septembre 2005, montrait ainsi que $34 \%$ des bénéficiaires du CLCA de rang 1 à taux partiel étaient professions intermédiaires, $25 \%$ ouvrières, $20 \%$ cadres et $16 \%$ employées. La part d'ouvrières et d'employées était supérieure parmi les bénéficiaires du CLCA à taux plein (respectivement $31 \%$ et $20 \%$ ). $55 \%$ des bénéficiaires du CLCA de rang 1 à taux réduit avaient, en septembre 2005, un niveau supérieur au bac, contre $42 \%$ des bénéficiaires à taux plein. En termes de revenus, $41 \%$ des premières disposaient de revenus très élevés, $31 \%$ de revenus élevés, et $20 \%$ de revenus moyens (Berger et al., 2006). Il semble donc que le CLCA de rang 1 ait modifié les comportements d'activité de toutes les mères (Ananian, 2010). Nous retrouvons bien cette évolution à partir des données sur les taux d'interruption par classes de salaire de la Génération $98^{7}$ avec, en particulier, des taux d'interruption à temps partiel nettement plus élevés après 2004 pour les mères dont les salaires correspondent aux tranches de salaires moyens à très élevés (figure 2).

7. Les classes de salaires sont découpées en quintiles. 
Tableau 4

Taux d'emploi et salaire moyen 12, 18 et 24 mois après la naissance du premier enfant

\begin{tabular}{|l|c|c|c|c|c|c|}
\hline Taux d'emploi & \multicolumn{3}{|c|}{ Naissance avant 2004 } & \multicolumn{3}{c|}{ Naissance après 2004} \\
\hline $\begin{array}{l}\text { T+ x mois après } \\
\text { la naissance }\end{array}$ & $\begin{array}{c}\text { Interruption à } \\
\text { taux plein }\end{array}$ & $\begin{array}{c}\text { Interruption à } \\
\text { taux partiel }\end{array}$ & Pas d'interruption & $\begin{array}{c}\text { Interruption à } \\
\text { taux plein }\end{array}$ & $\begin{array}{c}\text { Interruption à } \\
\text { taux partiel }\end{array}$ & $\begin{array}{c}\text { Pas } \\
\text { d'interruption }\end{array}$ \\
\hline$T+12$ & 35 & 82 & 87 & 83 & 98 & 94 \\
\hline$T+18$ & 46 & 87 & 87 & 84 & 96 & 92 \\
\hline$T+24$ & 35 & 87 & 88 & 85 & 96 & 90 \\
\hline
\end{tabular}

\begin{tabular}{|l|c|c|c|c|c|c|}
\hline $\begin{array}{l}\text { Salaire moyen } \\
\text { (en } € \text { ) }\end{array}$ & \multicolumn{3}{|c|}{ Naissance avant 2004 } & \multicolumn{3}{c|}{ Naissance après 2004} \\
\hline $\begin{array}{l}\text { T+x mois après } \\
\text { la naissance }\end{array}$ & $\begin{array}{c}\text { Interruption à } \\
\text { taux plein }\end{array}$ & $\begin{array}{c}\text { Interruption à } \\
\text { taux partiel }\end{array}$ & Pas d'interruption & $\begin{array}{c}\text { Interruption à } \\
\text { taux plein }\end{array}$ & $\begin{array}{c}\text { Interruption à } \\
\text { taux partiel }\end{array}$ & $\begin{array}{c}\text { Pas } \\
\text { d'interruption }\end{array}$ \\
\hline $\mathrm{T}+12$ & 461 & 995 & 1135 & 1032 & 1267 & 1365 \\
\hline $\mathrm{T}+18$ & 1223 & 1157 & 1304 & 1173 & 1268 & 1460 \\
\hline $\mathrm{T}+24$ & 1311 & 1184 & 1327 & 1165 & 1290 & 1468 \\
\hline
\end{tabular}

Source : 4 I Interrogation en 2008 de la Génération 1998, Céreq.

Champ : mères d'un premier enfant en emploi avant la naissance.

Note de lecture : $82 \%$ des mères d'un premier enfant né avant 2004 passées à temps partiel sont toujours actives un an après, contre $98 \%$ de celles ayant fait le même choix après la réforme.

\section{Impact de la réforme de la PAJE sur les carrières des mères}

La réforme de la PAJE, en proposant un congé court rémunéré dès le premier enfant, a incité plus de mères à prendre un congé parental. Quelles ont été les conséquences à plus long terme de ces interruptions sur les trajectoires d'emploi et de salaire des femmes ?

Pour répondre à cette question, nous observons le statut d'activité et le salaire des mères qui ont repris le travail au-delà de la période de prestation de l'allocation. La majorité des bénéficiaires du CLCA ne le percevant plus 12 mois après la naissance, nous étudions la trajectoire professionnelle des mères 12,18 et 24 mois après la naissance, en nous intéressant à la fois à leur statut d'activité (emploi/ chômage /inactivité) et à leur salaire.

\subsection{Une méthode d'évaluation}

Le taux d'emploi des femmes s'élève à $94 \%$ avant la naissance de leur premier enfant et chute à $88 \%$ un ou deux ans après. Ce taux a-t-il changé après la mise en place de la réforme de la PAJE ? Autrement dit, la réforme a-t-elle pu inciter des mères prenant un congé parental à s'arrêter de travailler au-delà de la période de prestation du CLCA ? (soit 6 mois). 


\section{Encadré 3}

\section{Méthode d'estimation pour identifier l'effet moyen du CLCA (complément libre choix d'activité pour premier enfant) sur les bénéficiaires}

Formellement, le problème est relativement simple à exposer. Considérons $Y_{i 1}$ la valeur du salaire $x$ mois après la naissance pour les mères ayant bénéficié du CLCA (le traitement) et $Y_{i 0}$ cette valeur lorsque les mères n'ont pas bénéficié du CLCA. Pour une même personne, il n'est possible d'observer qu'une seule réalisation de $Y$, alors que l'on souhaiterait savoir quelle aurait été la valeur de $Y$ si cette même personne avait connu l'autre situation. Notons $T_{i}$ l'indicateur d'accès au CLCA, que l'on appelle communément le traitement dans les méthodes quantitatives d'évaluation. $T_{i}$ prend la valeur 1 si la mère opte pour le CLCA, 0 sinon. L'évaluation du traitement qui est couramment proposée repose sur l'estimation de l'effet moyen du traitement sur les personnes traitées (les allocataires) (ATT), soit :

$$
A T T=E\left(Y_{i 1} \mid T_{i}=1\right)-E\left(Y_{i 0} \mid T_{i}=1\right)
$$

L'effet moyen du traitement sur les traitées, l'ATT, est estimé en comparant les différences moyennes des variables d'intérêt (ici le salaire et le taux d'activité) sur les individus traités. L'écriture de cet effet moyen de traitement sur les traités a toutefois une portée davantage conceptuelle, puisqu'il n'est pas possible d'observer $Y_{i 0}$ pour les individus satisfaisant à $T_{i}=1$. Ce n'est qu'en faisant l'hypothèse d'une sélection sur les caractéristiques observables que l'on peut identifier cet effet.

Il est alors nécessaire d'estimer $E\left(Y_{i} \mid X_{i}, T_{i}=1\right)$ et $E\left(Y_{i} \mid X_{i}, T_{i}=0\right)$ en utilisant en étape intermédiaire le score de propension tel que défini par Rosenbaum et Rubin (1983), obtenu par l'estimation d'une régression logistique de la probabilité de réduire l'activité professionnelle et de bénéficier du CLCA.

La principale hypothèse que l'on fait ici implique que l'assignation au traitement (le fait de bénéficier du dispositif) est indépendante conditionnellement à la variable d'intérêt (voir Heckman et Robb, 1985). En effet, sans cette hypothèse, on obtiendrait un estimateur biaisé de I'ATT puisqu'il existerait une sélection sur les variables observables, qui déterminerait des valeurs particulières de $Y$ pour chacun des groupes. Cette hypothèse fonde toute la méthode d'appariement. Elle permet d'utiliser le groupe des non-traités ayant une distribution similaire des caractéristiques observables; il s'agit de mesurer ce que les bénéficiaires auraient comme salaire si elles n'avaient pas interrompu leur activité en contrepartie d'une indemnité.

En pratique, plusieurs méthodes d'appariement sont possibles. La plus simple consiste à associer à chaque individu i ayant perçu le CLCA un individu non traité, dont les caractéristiques $\mathrm{X}$ sont identiques à celles de l'individu i. L'inconvénient est que dès que le nombre de variables $X$ est trop important (ou si certaines d'entre elles sont continues), l'appariement devient difficile. Dès lors, Rosenbaum et Rubin (1983) proposent d'apparier les individus non pas sur les caractéristiques $\mathrm{X}$ mais sur une fonction de ces variables, appelée score de propension. Plusieurs algorithmes peuvent être utilisés pour choisir le ou les individus jumeaux qui ont des scores de propension proches des individus séparés. On peut prendre le ou les plus proches voisins ( $" \mathrm{k}$ nearest-neighbour method »), minimiser une fonction de la somme des distances entre jumeaux et bénéficiaires du congé parental (distance de Mahalanobis) ou encore utiliser un estimateur à noyau (voir Afsa et Givord, 2009). Ce dernier, que nous choisissons, consiste à retenir, pour chaque individu ayant pris un congé parental, tous les individus ne l'ayant pas pris, en les affectant d'un poids inversement proportionnel à leur distance avec l'individu ayant pris un congé parental. 
Les taux d'emploi des mères ayant connu une interruption à taux partiel restent très élevés après la naissance de leur premier enfant (tableau 4) et bien plus élevés qu’avant la réforme. Ce taux reflète la composition de ce groupe, composé en majorité des mères diplômées de l'enseignement supérieur et bénéficiant de salaires moyens à très élevés. En revanche, si leur taux d'emploi est parmi les plus élevés, leur niveau de salaire moyen reste inférieur à celui des mères n'ayant pas connu d'interruption de carrière. Pourrait-on y lire un effet de cette interruption et, par là, un effet induit de la réforme ayant incité plus de mères à interrompre partiellement leur activité ?

Etudier l'effet de la réforme sur la reprise ou le maintien dans l'activité implique de prendre en compte la manière dont l'activité des mères qui prennent un congé parental aurait évolué en l'absence de réforme. Or, la prise d'un congé parental lors de la naissance d'un premier enfant est un événement unique (on ne peut l'observer simultanément avant et après la réforme pour un même individu); on ne peut donc pas observer directement l'effet de la réforme sur les mêmes mères. Il convient donc de comparer le comportement sur le marché du travail des femmes qui ont pris un congé parental après la réforme, appelées les " traitées " (ou allocataires), à celles qui l'auraient pris avant 2004 si la mesure avait été alors disponible. On appelle ce dernier groupe le contrefactuel ou le groupe de contrôle.

On pourrait comparer directement les deux groupes, mais la structure des femmes qui prennent un congé parental est particulière. Il est donc nécessaire de trouver un échantillon "témoin ": on cherche, parmi les femmes devenues mères avant 2004, un groupe de femmes comparable à celui des mères ayant bénéficié de la réforme lors de la naissance de leur premier enfant. L'hypothèse implicite est que l'on dispose de deux populations comparables dont l'une a bénéficié de la réforme et l'autre non. Les éventuelles différences observées sont alors imputables à la mise en place de la réforme. Les méthodes d'appariement (voir Brodaty et al. 2007 ; Givord, 2010 ; Bonnet et al., 2010) sont bien adaptées pour construire ce groupe témoin en fonction des caractéristiques observables ${ }^{8}$ (encadré 3).

Afin d'apparier les individus, nous estimons la probabilité de prendre un congé parental (score de propension) sur les variables suivantes : le niveau d'éducation, le salaire 12 mois avant la naissance, le statut du conjoint à la naissance, l'inactivité de la propre mère de l'enquêtée à la fin des études, le niveau de diplôme et la région de résidence. On vérifie que les distributions des deux populations se recouvrent partiellement afin d'assurer que l'on trouvera facilement pour toute femme ayant pris un CLCA, une femme comparable avant 2004 avec un score de propension similaire.

8. Il est aussi possible de tenir compte des caractéristiques inobservables en combinant un estimateur de différences de différences avec la procédure d'appariement (Heckman et al., 1997). 
Tableau 5

Effet moyen du CLCA (complément libre choix d'activité pour premier enfant) pour les bénéficiaires

\begin{tabular}{|c|c|c|c|c|c|}
\hline & $\begin{array}{l}\text { Groupe des } \\
\text { "traitées » } \\
\text { (bénéficiaires } \\
\text { du CLCA) }\end{array}$ & $\begin{array}{l}\text { Groupe de } \\
\text { « contrôle » } \\
\text { (les éligibles } \\
\text { avant 2004) }\end{array}$ & Différence & Ecart-type & $\begin{array}{c}\text { Significativité } \\
\text { (statistique } \\
\text { de Student) }\end{array}$ \\
\hline \multicolumn{6}{|c|}{ Activité après la naissance } \\
\hline \multicolumn{6}{|c|}{$\mathrm{T}+12$ mois } \\
\hline En emploi & 96,4 & 94,5 & $1,8^{*}$ & 1,2 & 1,83 \\
\hline Au chômage & 2,9 & 0,3 & 0,4 & 0,6 & $-0,44$ \\
\hline Inactive & 0,7 & 0,2 & $-1,4^{* *}$ & 0,6 & $-2,33$ \\
\hline \multicolumn{6}{|c|}{$\mathrm{T}+18$ mois } \\
\hline En emploi & 96,7 & 94,3 & $2,5^{* *}$ & 1,1 & 2,20 \\
\hline Au chômage & 2,7 & 3,3 & $-0,5$ & 1,0 & $-0,55$ \\
\hline Inactive & 0,5 & 2,5 & $-1,9^{* * *}$ & 0,6 & $-3,04$ \\
\hline \multicolumn{6}{|c|}{$\mathrm{T}+24$ mois } \\
\hline En emploi & 94,3 & 93,7 & 0,6 & 1,5 & 0,39 \\
\hline Au chômage & 3,6 & 3,0 & 0,6 & 1,2 & 0,47 \\
\hline Inactive & 2,1 & 3,3 & $-1,2$ & 0,9 & $-1,30$ \\
\hline \multicolumn{6}{|c|}{ Salaire après la naissance (pour celles en emploi) } \\
\hline$T+12$ mois & 1340 & 1414 & $-74^{* * *}$ & 19,02 & $-3,88$ \\
\hline $\mathrm{T}+18$ mois & 1331 & 1406 & $-75^{* * *}$ & 22,17 & $-3,36$ \\
\hline$T+24$ mois & 1341 & 1440 & $-99^{* * *}$ & 24,47 & -4.03 \\
\hline
\end{tabular}

Source : $4^{e}$ Interrogation de la Génération 1998, Céreq.

Champ : mères d'un premier enfant en emploi avant la naissance.

${ }^{* * *}$ significatif au seuil de $1 \%,{ }^{* *}$ significatif au seuil de $5 \%,{ }^{*}$ significatif au seuil de $10 \%$.

Note de lecture : $96,4 \%$ des bénéficiaires du CLCA ont repris un emploi un an après la naissance contre seulement $94,5 \%$ de la population de contrôle. La différence de taux est significative au seuil de $10 \%$.

L'effet de la réforme de la PAJE sur la trajectoire professionnelle des femmes est obtenu en calculant la moyenne empirique des écarts de taux d'emploi et de salaires entre le groupe d'allocataires du CLCA et le groupe contrefactuel. Il est cependant nécessaire de recalculer l'écart-type de cet estimateur par la méthode du bootstrap (ici, 100 itérations).

\subsection{La réforme de la PAJE affecte davantage les salaires que le taux d'activité}

La part de femmes en emploi un an après la naissance est similaire pour les mères allocataires du CLCA et leurs contrefactuelles : elle s'élève respectivement à 96,4 \% et 94,5\%, la différence n'étant pas significative (tableau 5). La réforme de la PAJE pour le premier enfant n'a donc pas réduit l'activité professionnelle des mères au-delà de la période de perception de l'allocation. À l'inverse, on constate un moindre passage vers l'inactivité 
Tableau 6

Effet moyen du CLCA (complément libre choix d'activité premier enfant) à taux plein sur les bénéficiaires

\begin{tabular}{|c|c|c|c|c|c|}
\hline & $\begin{array}{l}\text { Groupe des } \\
\text { «traitées » } \\
\text { (bénéficiaires } \\
\text { du CLCA } \\
\text { à taux plein) }\end{array}$ & $\begin{array}{l}\text { Groupe de } \\
\text { " contrôle » } \\
\text { (les éligibles } \\
\text { avant 2004) }\end{array}$ & Différence & Ecart-type & $\begin{array}{c}\text { Significativité } \\
\text { (statistique } \\
\text { de Student) }\end{array}$ \\
\hline \multicolumn{6}{|c|}{ Activité après la naissance } \\
\hline \multicolumn{6}{|c|}{$\mathrm{T}+12$ mois } \\
\hline En emploi & 90,8 & 92,4 & $-1,6$ & 2,3 & $-0,70$ \\
\hline Au chômage & 7,2 & 4,6 & 2,6 & 2,2 & 1,18 \\
\hline Inactive & 1,9 & 30,1 & $-1,0$ & 1,4 & $-0,75$ \\
\hline \multicolumn{6}{|c|}{$T+18$ mois } \\
\hline En emploi & 92,6 & 92,6 & 0,0 & 2,4 & 0,00 \\
\hline Au chômage & 5,9 & 03,9 & 1,9 & 2,1 & 0,94 \\
\hline Inactive & 1,5 & 3,5 & $-2,0$ & 1,5 & $-1,36$ \\
\hline \multicolumn{6}{|c|}{$T+24$ mois } \\
\hline En emploi & 90,5 & 91,8 & $-1,3$ & 2,8 & $-0,48$ \\
\hline Au chômage & 7,1 & 3,6 & 3,5 & 2,5 & 1,43 \\
\hline Inactive & 2,4 & 4,6 & $-2,2$ & 1,6 & $-1,41$ \\
\hline \multicolumn{6}{|c|}{ Salaire après la naissance (pour celles en emploi) } \\
\hline$T+12$ mois & 1291 & 1300 & -8.47 & 23,77 & $-0,36$ \\
\hline$T+18$ mois & 1270 & 1269 & 0,42 & 30.42 & 0,01 \\
\hline $\mathrm{T}+24$ mois & 1289 & 1334 & -45 & 33,83 & $-1,33$ \\
\hline
\end{tabular}

Source : $4^{e}$ Interrogation de la Génération 1998, Céreq.

Champ : mères d'un premier enfant en emploi avant la naissance.

*** significatif au seuil de $1 \%$, ${ }^{* *}$ significatif au seuil de $5 \%$, ${ }^{*}$ significatif au seuil de $10 \%$.

Note de lecture : 90,8\% des bénéficiaires du CLCA à taux plein ont repris un emploi un an après la naissance contre 92,4\% pour la population de contrôle. La différence de taux n'est pas significative.

pour les mères qui ont bénéficié du CLCA pour le premier enfant, par rapport à celles qui auraient pu en bénéficier avant 2004. Ce résultat est cependant à nuancer car il repose sur des effectifs faibles. Il pourrait montrer qu'une catégorie des mères souhaite s'arrêter audelà du congé de maternité. Ces mères basculaient dans l'inactivité avant 2004 ; la réforme leur a permis de prendre un congé plus long et de retourner au travail à l'issue de celui-ci. Ainsi, pour une première naissance, contrairement aux congés parentaux plus longs qui ont tendance à éloigner durablement les mères du marché du travail (Piketty, 2005), ce type de congé plus court a moins d'effets pervers sur l'activité féminine. Nous ne sommes pas en mesure d'estimer si cet effet reste valide pour les naissances de rang supérieur. Or la réduction de la participation au marché du travail (passage à temps partiel ou inactivité) s'amplifie au fil des naissances (Pailhé et Solaz, 2006). 
Tableau 7

Effet moyen du CLCA (complément libre choix d'activité au premier enfant) à taux réduit pour les bénéficiaires

\begin{tabular}{|c|c|c|c|c|c|}
\hline & $\begin{array}{l}\text { Groupe des } \\
\text { " traitées » } \\
\text { (bénéficiaires } \\
\text { du CLCA } \\
\text { à taux réduit) }\end{array}$ & $\begin{array}{l}\text { Groupe de } \\
\text { " contrôle » } \\
\text { (les éligibles } \\
\text { avant 2004) }\end{array}$ & Différence & Ecart-type & $\begin{array}{c}\text { Significativité } \\
\text { (statistique } \\
\text { de Student) }\end{array}$ \\
\hline \multicolumn{6}{|c|}{ Activité après la naissance } \\
\hline \multicolumn{6}{|c|}{$T+12$ mois } \\
\hline En emploi & 99,6 & 95,4 & $4,2^{* * *}$ & 0,8 & 5,19 \\
\hline Au chômage & 0,4 & 2,7 & $-2,3^{* * *}$ & 0,6 & $-3,84$ \\
\hline Inactive & 0 & 1,9 & $-1,9 * * *$ & 0,5 & $-4,05$ \\
\hline \multicolumn{6}{|c|}{$T+18$ mois } \\
\hline En emploi & 98,7 & 95,1 & $3,6^{* * *}$ & 1,1 & 3,21 \\
\hline Au chômage & 0,9 & 2,8 & $-1,9^{* *}$ & 0,9 & $-2,20$ \\
\hline Inactive & 0,4 & 2,1 & $-1,6^{* *}$ & 0,7 & $-2,28$ \\
\hline \multicolumn{6}{|c|}{$T+24$ mois } \\
\hline En emploi & 96,7 & 94,8 & 1,8 & 1,4 & 1,25 \\
\hline Au chômage & 1,4 & 2,5 & $-1,1$ & 0,9 & $-1,20$ \\
\hline Inactive & 1,9 & 2,7 & $-0,7$ & 1,1 & $-0,68$ \\
\hline \multicolumn{6}{|c|}{ Salaire après la naissance (pour celles en emploi) } \\
\hline$T+12$ mois & 1376 & 1479 & $-103^{* * *}$ & 21,20 & $-4,89$ \\
\hline $\mathrm{T}+18$ mois & 1359 & 1473 & $-114^{* * *}$ & 24,66 & $-4,65$ \\
\hline$T+24$ mois & 1366 & 1503 & $-137^{* * *}$ & 33,13 & $-4,16$ \\
\hline \multicolumn{6}{|c|}{ Différence de salaires } \\
\hline$(T+12)-(T-12)$ & $-74,56$ & 53,21 & $-127,77^{* * *}$ & 14,14 & $-9,03$ \\
\hline
\end{tabular}

Source : $4^{e}$ Interrogation de la Génération 1998, Céreq.

Champ : mères d'un premier enfant en emploi avant la naissance.

*** significatif au seuil de $1 \%,{ }^{* *}$ significatif au seuil de $5 \%,{ }^{*}$ significatif au seuil de $10 \%$.

Note de lecture : $99,6 \%$ des bénéficiaires du CLCA à taux réduit ont repris un emploi un an après la naissance contre seulement 95,4\% de la population de contrôle. La différence de taux est significative au seuil de $1 \%$.

Quant aux salaires des mères qui ont repris une activité après la naissance et qui ont bénéficié du CLCA, ils sont inférieurs, à 12 et 18 mois, à ceux des mères ayant un même profil et qui en auraient bénéficié avant 2004 si la réforme avait été disponible. Cette moindre augmentation de salaires peut être due à l'interruption courte (dépréciation du capital humain pendant le congé de maternité rallongé, moindre promotion, signal négatif pour l'employeur) ; elle peut aussi résulter de passages à temps partiel qui se seraient prolongés au-delà de la période de perception du CLCA. Les données ne nous permettent malheureusement pas de savoir si ces mères sont effectivement passées à temps partiel. En revanche, il est possible de distinguer, parmi celles ayant eu recours au CLCA, celles qui l'ont pris à temps partiel. 
Pour évaluer si le CLCA a eu des effets différenciés sur l'activité et le salaire des mères selon qu'il a été pris à temps plein ou à temps partiel, l'étape suivante consiste à apparier des mères ayant eu recours au CLCA selon les deux modalités possibles : à temps complet avec interruption totale du travail, et à temps partiel (perception de l'allocation réduite tout en conservant un travail à temps partiel).

Pour les mères ayant interrompu totalement leur activité, on ne constate pas de différences tant dans la trajectoire d'activité, hormis la moindre probabilité d'être inactive déjà observée pour l'ensemble des bénéficiaires, que pour le profil de salaires post-naissance (tableau 6). La perception de l'allocation du CLCA n'a donc pas eu d'effet.

Pour les mères qui ont choisi de percevoir l'allocation réduite et de travailler à temps partiel, nous constatons de moindres chances de devenir inactives ou chômeuses et, symétriquement, de plus grandes chances de conserver un emploi à 12 et 18 mois (tableau 7). Continuer à travailler après la naissance protège du risque de chômage et d'inactivité en maintenant le lien avec l'emploi. Au bout de deux ans, ces différences s'estompent.

Si le maintien dans l'activité se poursuit, en revanche, le profil de salaires differe pour celles ayant choisi d'exercer à temps partiel. On constate ainsi de moindres salaires dans le groupe des " traitées " (allocataires) que dans le groupe de " contrôle ", et l'écart s'amplifie avec la durée écoulée depuis la naissance. Plusieurs explications peuvent être avancées. Il est possible que les mères ayant peu de perspective d'évolution de salaire (salariées au salaire minimum par exemple) aient profité de cette réforme pour opter pour un travail à temps partiel. Un effet d'aubaine existe sans doute aussi : les mères qui auraient pris de toute manière un temps partiel avant la réforme l'ont pris ici et ont bénéficié de l'allocation en plus. C'est le cas sans doute de mères moins carriéristes, avec des salaires moins élevés. Enfin, ces salaires moindres peuvent être liés à un travail à temps partiel qui se prolonge au-delà de la durée de perception de l'allocation. Dans ce cas probable, la réforme de la PAJE, en incitant les mères à passer à temps partiel (pour celles qui travaillent à $80 \%$ par exemple, la perte de salaire peut être complètement compensée par le montant de l'allocation), aurait donné goût à cette organisation familiale plus souple. Elles sont alors restées à temps partiel au-delà de la période de prestation. Il est enfin possible aussi que les projets d'un second enfant aient contribué à ce maintien à temps partiel.

L'appariement porte sur un ensemble de caractéristiques observables supposées capter les principales différences d'accès au congé parental entre les mères ; néanmoins, il subsiste probablement des caractéristiques inobservées, possiblement corrélées avec la variable d'intérêt (le salaire ou l'activité). Cela peut constituer une limite de ce type d'estimateurs (Dias, Ichimura, Van den Berg, 2008). Pour vérifier cela, nous avons associé un estimateur de différence de différence à cet appariement, en calculant la différence de salaires 12 mois avant et 12 mois après la naissance pour le groupe de "traitées » (allocataires) et de " contrôle" (tableau 7), comme suggéré par Guo et Fraser (2010). De manière générale, les estimateurs par différence éliminent la corrélation entre le traitement et la variable d'intérêt par différenciation des données observées avec celles du groupe de contrôle (Crépon 
et Jacquemet, 2010). Pour les bénéficiaires du CLCA, la différence de salaire reste significative et négative une fois prise en compte l'hétérogénéité inobservée. Pour les allocataires du CLCA à taux réduit, on observe un différentiel de salaire négatif; cela confirme l'hypothèse de maintien à temps partiel au-delà de la durée de perception de l'allocation.

\section{Conclusion}

Cette étude cherche à évaluer l'impact de l'introduction, en 2004, du complément libre choix d'activité (CLCA), mis en place par la Cnaf dans le cadre de la prestation d'accueil du jeune enfant (PAJE). Elle visait à aider financièrement les jeunes parents en contrepartie d'une interruption momentanée de leur activité professionnelle lors de la naissance de leur premier enfant. Avant 2004, les jeunes femmes pouvaient opter pour un congé parental, mais sans aucune forme d'indemnisation financière compensatrice. Cette réforme de 2004, en élargissant le public des bénéficiaires, malgré les conditions d'éligibilité au dispositif, a permis à plus de jeunes mères d'interrompre leur activité professionnelle après la naissance de leur premier enfant. Alors que jusque-là, la politique familiale ciblait la majorité de ses aides sur les enfants de rang supérieur à deux, le choix d'indemniser un congé parental court de six mois pour la naissance du premier enfant relance le débat autour de l'interruption d'activité des femmes dans les périodes de maternité.

Notre travail s'inscrit dans une démarché complémentaire à celle de Lequien (2012), visant à proposer une évaluation rigoureuse de ce nouveau dispositif. S'appuyant sur les données d'enquête de la $4^{\mathrm{e}}$ interrogation de la Génération 98 du Céreq, nous montrons d'une part, que les jeunes mères interrompent plus fréquemment leur activité entre 2004 et 2008, comparativement à la période 2000-2004; d'autre part, que cette interruption est le plus souvent à temps partiel pour les mères les plus diplômées ayant accédé à des positions professionnelles élevées. Si le congé parental à plein temps n'a aucun effet sur la participation au marché du travail et sur les salaires, les résultats diffèrent pour le congé pris à temps partiel. Ils suggèrent que les mères s'habituent à travailler à temps partiel et le restent au-delà de la période de perception de l'allocation.

Il ressort donc qu'un congé parental court a moins d'effets négatifs sur la participation féminine au marché du travail qu'un congé long. La formule à temps partiel a en effet remporté plus de succès auprès des mères au salaire moyen ou élevé, tandis que le bas de la distribution des revenus a opté pour un congé parental à taux plein ; cela laisse penser que la polarisation sociale reste forte. On peut y voir un effet d'aubaine pour les mères au revenu intermédiaire ; pour ces dernières en effet, la perte de salaire liée au passage à temps partiel à $80 \%$ peut être quasiment compensée par la prestation. Le débat sur la mise en place d'un congé parental court mais mieux indemnisé reste donc à l'ordre du jour. Il pourrait contribuer à gommer ces effets de polarisation sociale. En effet, une meilleure indemnisation serait susceptible de moins pénaliser les jeunes femmes souhaitant mettre une parenthèse, même brève, à leur activité professionnelle et aussi à inciter les pères à s'arrêter. 


\section{Bibliographie}

Afsa C., Givord P. (2009), "The impact of working conditions on absenteeism: a theoretical model and an application to work schedules", WP Crest n ${ }^{\circ}$ 2009-06.

Albrecht J., Edin P.A., Sundström M., Vroman S. (1999), "Career interruptions and subsequent earnings: a reexamination using Swedish data", The Journal of Human Resources, 34(2), pp. 294-311.

Ananian S. (2010), « Lactivité des mères de jeunes enfants depuis la mise en place du complément libre choix d'activité », Etudes et Résultats, Drees, nº 726.

Becker G. (1964), Human Capital. Theoretical and Empirical Analysis, with Special Reference to Education. Chicago, University of Chicago Press.

Berger E., Chauffaut D., Olm C., Simon M. O. (2006), " Les bénéficiaires du Complément de libre choix d'activité : une diversité de profils ", Etudes et Résultats, Drees, $\mathrm{n}^{\circ} 510$.

Bonnet C., Solaz A., Algava E. (2010), "Les changements professionnels en France autour de la séparation conjugale ", Population, avril-juin, vol. 65, n 2, pp. 273-308.

Brodaty T., Crépon B., Fougère D. (2007), "Les méthodes micro-économétrique d'évaluation et leurs applications aux politiques actives de l'emploi ", Economie et Prévision, 177(1).

Crépon B., JaCquemet N. (2010), Econométrie : méthode et applications, De Boeck Université.

Dias M.C., Ichimura H., Van den Berg G.J. (2008), "The matching method for treatment evaluation with selective participation and ineligibles", IZA DP, $\mathrm{n}^{\circ} 3280$.

HCF Haut Conseil de la Famille (2009), Les aides apportées aux familles qui ont un enfant de moins de trois ans. Note du HCF : http://www.hcf-famille.fr/IMG/pdfIHCF_note_ jeunes_enfants_V_du_03122009.pdf

Grvord P. (2010), "Econometric methods for public policies evaluation", Document de travail de la DESE, Insee, G2010-08.

Grésy B., Dole P., Снivot F. (2011), L'égal accès des femmes et des hommes aux responsabilités professionnelles et familiales dans le monde du travail, rapport de l'IGAS.

Guo S., Fraser M.W. (2010), Propensity score analysis : statistical methods and applications, Sage eds.

Heckman J.J., Ichimura H., Todd P. (1997), "Matching as an econometric evaluation estimator”, Review of Economic Studies, 64, pp. 605-654. 
Heскмan J.J., Rовв R. (1985), "Alternative methods for evaluating the impact of interventions", in Heckman J., and Singer B., (eds.), Longitudinal analysis of Labor Market Data, Cambridge University Press.

Jaumotte F. (2003), "Female labour force participation: past trends and main determinants in OECD countries", OECD Economics Department Working Papers, n 376.

LEQUien L. (2012), "L'impact sur les salaires de la durée d'une interruption de carrière suite à une naissance ", Annals of Economics and Statistics, à paraitre.

Meurs D., Pailhé A., Ponthieux S. (2011), "Child-related career interruptions and the gender wage gap in France", Annals of economics and statistics, 99/100, pp. 15-46.

Mincer J., Polacher S. (1974), "Family investments in human capital: earnings of women”, Journal of Political Economy Vol. 82, n 2 Part II, pp. S76-S108.

Nicolas M. (2010), «Interrompre ou réduire son activité à la naissance d'un enfant, et bénéficier du CLCA de la PAJE ", l'e-ssentiel, CNAF, nº 97.

Pailhe A., Solaz A. (2006), "Vie professionnelle et naissance : la charge de la conciliation repose essentiellement sur les femmes ", Population et Sociétés, 436.

OCDE (2011), Assurer le bien-être des familles, Ocde, Paris.

PiketTy T. (2005), «L'impact de l'allocation parentale d'éducation sur l'activité féminine et la fécondité en France, 1982-2002 ", in Lefèvre C. (Ed.), Histoires de familles, histoires familiales, Les Cahiers de l'INED, n 156, pp. 79-109.

Recotillet I., Rouaud P., Ryk F. (2011), « Regards sur les dix premières années de vie active d'une génération ", NEF-Céreq, $\mathrm{n}^{\circ} 45$.

Rosenbaum P., Rubin D. (1983), "The central role of the propensity score in observational studies for causal effects", Biometrika, 70, 1, pp. 41-55.

Ruнm C. (1998), "The Economic consequences of parental leave mandates: Lessons from Europe", The Quarterly Journal of Economics, 113(1), pp. 285-317. 